\title{
DISCRIMINATORY SOLUTIONS OF $n$-PERSON GAMES ${ }^{1}$
}

\section{GUILLERMO OWEN}

In studying $n$-person games, we find that very often solutions exist in which one, or several, of the players, always receive the same amount. Such players are said to be discriminated players, and the solutions are said to be discriminatory. It is our purpose to study these solutions.

Definition 1. Let $v$ be an $n$-person game, let $S$ be a subset of $N$ (the set of all players) and let $q \leqq v(N)$. Then by $\bar{v}_{S, q}$ we mean the game defined by

$$
\bar{v}(T)= \begin{cases}v(T) & \text { if } T \subset S \text { but } T \neq S, \\ q & \text { if } T=S .\end{cases}
$$

In general, $\bar{v}$ will not be a super-additive function and hence, strictly speaking, is not a game. However, imputations, domination, and solutions can be defined for $\bar{v}$ just as for any super-additive function.

Definition 2. In a game $v$, a coalition $S$ is effective for $q$ if and only if $v(S) \geqq q$.

Definition 3. In a game $v$, a coalition $S$ is strong for $q$ if and only if the core of the game $\bar{v}_{S, q}$ has no interior points.

LeMma 1. Let $v$ be an n-person game and let $V$ be a discriminatory solution, discriminating against the members of $N-S$, who receive the amounts $\alpha_{j}$. Then the restriction of $V$ to $S$ (the set of s-vectors which are the $S$-components of elements of $V$ ) is a solution to $\bar{v}_{S, q}$ where

$$
q=v(N)-\sum_{j \in N-S} \alpha_{j}
$$

Proof. Let $x$ and $y$ be two elements of $V$. It is not possible that the $S$-part of $x$ dominate that of $y$ in $\bar{v}_{S, q}$, as this domination would be through a proper subset $T$ of $S$, for which $\bar{v}(T)=v(T)$ and this would mean $x \varepsilon y$ in $v$.

Similarly, let $z$ be an imputation in $\bar{v}$ which is not the $S$-part of some element of $V$. Adding the components $\alpha_{j}$ (for $j \in N-S$ ) to $z$, we obtain an imputation for $v$. This is not in $V$, hence is dominated by some $x \in V$. But this must be through some proper subset $T$ of $S$.

Received by the editors July 17, 1964.

1 The research for this paper was supported in part by a grant from the National Science Foundation. 
( $T$ cannot include elements of $N-S$, as for these, $x_{j}=\alpha_{j}=z_{j}$, nor can it be $S$, as $\sum_{j \in S} x_{j}=\sum_{j \in S} z_{j}$.) But $\bar{v}(T)=v(T)$, so the $S$-part of $x$ dominates $z$ in $\bar{v}$.

Lemma 2. Let $v$ be an $n$-person game, $S$ a subset of $N$, and $q \leqq v(N)$. Let $\alpha$ be an $(n-s)$-vector defined on $N-S$ such that $\sum_{j \in N-S} \alpha_{j}=v(N)$ $-q$. Let $\bar{V}$ be a solution to $\bar{v}_{S, q}$ and let $V^{*}$ be obtained from $\bar{V}$ by adding the components $\left(\alpha_{j}\right)$ to all elements of $\bar{V}$. Then the elements of $V^{*}$ will dominate all imputations $y$ of $v$ for which

$$
\sum_{j \in N-S} y_{j}>\sum_{j \in N-S} \alpha_{j}
$$

if and only if $S$ is either effective or strong for $q$.

Proof. Sufficiency: Let $y$ be any such imputation of $v$. Then

$$
\sum_{j \in N-S}\left(y_{j}-\alpha_{j}\right)=\epsilon>0 .
$$

Consider the imputation $z$ defined by

$$
z_{j}=\left\{\begin{array}{l}
\alpha_{j} \text { if } j \in N-S, \\
y_{j}+\epsilon / s \text { if } j \in S .
\end{array}\right.
$$

Then the $S$-part of $z$ is an imputation in $\bar{v}_{S, q}$, and hence, either $z_{S} \in \bar{V}$, or there exists $x_{S} \in \bar{V}$ such that $x_{S} \varepsilon z_{S}$ in $\bar{v}$.

Suppose $z_{S} \in \bar{V}$. Then, if $S$ is effective for $q, z \varepsilon y$ (in $v$ ) through the set $S$. If $S$ is strong for $q$, then there is some $T \subset S$ such that $v(T)$ $\geqq \sum_{j \in T} z_{j}$. Hence $z \varepsilon y$ through the set $T$.

Suppose $z_{S} \notin \bar{V}$. Then $x_{S} \in \bar{V}$, and if $x_{S} \varepsilon z_{S}$ through a set $T$ (which must be a proper subset of $S$ ) then $x \varepsilon y$ (in $v$ ) through this same set $T$, as $\bar{v}(T)=v(T)$.

Necessity. Suppose $S$ is neither strong nor effective for $q$. This means there is an imputation $z_{S}$ in $\bar{v}$ such that, for every $T \subset S, v(T)<\sum_{j \in T} z_{j}$. Thus, there is $\epsilon>0$ such that, for every $T \subset S$

$$
v(T) \leqq \sum_{j \in T}\left(z_{j}-\epsilon\right) .
$$

Consider, then an imputation $y$ of $v$ such that $y_{j}=z_{j}-\epsilon / 2$ for all $j \in S$, and $y_{j}>\alpha_{j}$ for $j \in N-S$. Then, if $x \in V^{*}$, we find $x$ cannot dominate $y$. For, this domination must be through a set $T$ which cannot be a subset of $S$, for in such a case $v(T)<\sum_{j \in T} y_{j}$, nor can it include members of $N-S$ as $y_{j}>x_{j}$ for $j \in N-S$.

A third lemma would be useful here, stating under which condi- 
tions the set $V^{*}$ would dominate the imputations of $v$ other than those of $V^{*}$ itself and those covered by Lemma 2 . However, while a lemma can be stated, it would not really say much. The following observations are useful, and more to the point.

For any $y \notin V^{*}$, not covered by Lemma 2 the question is whether it will be dominated by some $x \in V^{*}$. Now, for $j \in N-S$, there are some $j$ such that $y_{j}<\alpha_{j}$ and some such that $y_{j} \geqq \alpha_{j}$. To check whether $V^{*}$ is a solution, we should try to make $y$ as difficult to dominate as possible. This is done by letting $y_{j}=v(\{j\})$ whenever $y_{j}<\alpha_{j}$, and $y_{j}=\alpha_{j}$ whenever $y_{j} \geqq \alpha_{j}$. Since $z_{j}=\alpha_{j}$ for all $z \in V^{*}$, this allows us to make $y_{j}$ as large as possible for $j \in S$, without making any more of the inequalities $z_{j}>y_{j}$ (for $j \in N-S$ ) true than is necessary.

Application.

Doubly Discriminatory Solutions of 4-Person Games. A game is doubly discriminatory if there are two discriminated players.

Let $v$ be a 4 -person game in $(0,1)$ normalization. Let $\alpha_{3} \geqq \alpha_{4} \geqq 0$, $\alpha_{3}+\alpha_{4} \leqq 1$. We would like to know whether $v$ has any solutions in which players 3 and 4 receive constantly $\alpha_{3}$ and $\alpha_{4}$ respectively.

Suppose that $V$ is such a solution. Then the set of $\{1,2\}$-components of $V$ must be a solution to the game $\bar{v}_{S, q}$ where $S=\{1,2\}$ and $q=1-\alpha_{3}-\alpha_{4}$. But this game $\bar{v}$ has a unique solution consisting of all its imputations. Hence $V$ must consist of all vectors $\left(x_{1}, x_{2}, \alpha_{3}, \alpha_{4}\right)$ where $x_{1} \geqq 0, x_{2} \geqq 0, x_{1}+x_{2}=q$.

Next, the set $\{1,2\}$ must be either effective or strong for $q$. But a two-person set is never strong, hence we must have

$$
v(\{1,2\}) \geqq q .
$$

Now we must consider whether $V$ dominates imputations $y$ such that $y_{3}+y_{4} \leqq \alpha_{3}+\alpha_{4}$. We consider the cases (i) $y_{3}=0, y_{4}=\alpha_{4}$; (ii) $y_{3}$ $=\alpha_{3}, y_{4}=0$; (iii) $y_{3}=y_{4}=0$.

In Case (i), if $\alpha_{3}>0, y \notin V$, and must be dominated through either $\{1,3\}$ or $\{2,3\}$. So that it be not dominated through $\{1,3\}$ by any $x \in V$, we must have

$$
y_{1} \geqq \min \left\{q, v(\{1,3\})-\alpha_{3}\right\} .
$$

So that it be not dominated through $\{2,3\}$ by any $x \in V$, we must have

$$
y_{2} \geqq \min \left\{q, v(\{2,3\})-\alpha_{3}\right\} .
$$

So that no such $y$ exists, it is necessary that

$$
v(\{1,3\})+v(\{2,3\})-2 \alpha_{3}>1-\alpha_{4} .
$$


If $\alpha_{3}=0$, then $y \in V$ and condition (II) becomes unnecessary.

In Case (ii), if $\alpha_{4}>0$ then similar reasoning to the above gives us

$$
v(\{1,4\})+v(\{2,4\})-2 \alpha_{4}>1-\alpha_{3} .
$$

If $\alpha_{4}=0$, then $y \in V$ and condition III is unnecessary.

If both $\alpha_{3}$ and $\alpha_{4}$ are positive, then conditions II and III must hold simultaneously. Adding them, we obtain

$$
v(\{1,3,\})+v(\{2,4\})+v(\{1,4\})+v(\{2,3\})>2+\alpha_{3}+\alpha_{4} .
$$

But $v(\{1,3\})+v(\{2,4\}) \leqq 1$, so the left-hand side in IV is smaller than 2 and hence IV is impossible. Thus, it is not possible that $\alpha_{3} \geqq \alpha_{4}>0$, so assuming $\alpha_{3} \geqq \alpha_{4}$, then $\alpha_{4}=0$.

Case (ii), then is meaningless, and Case (iii) reduces to Case (i), so we have

THEOREM. The four-person game v has a doubly discriminatory solution $V$ in which players 3 and 4 get $\alpha_{3}$ and $\alpha_{4}$ respectively, with $\alpha_{3} \geqq \alpha_{4}$, if and only if:

(a) $\alpha_{4}=0$

(b) $v(\{1,2\}) \geqq 1-\alpha_{3}$;

(c) If $\alpha_{3}>0$, then $v(\{1,3\})+v(\{2,3\})>1+2 \alpha_{3}$.

In this case $\mathrm{V}$ will consist of all imputations $\left(x_{1}, x_{2}, \alpha_{3}, 0\right)$, where $x_{1} \geqq 0, x_{2} \geqq 0, x_{1}+x_{2}=1-\alpha_{3}$. Setting (b) in (c), we obtain

COROLlary. The game $v$ will have such a solution if and only if either $v(\{1,2\})=1$, or $2 v(\{1,2\})+v(\{1,3\})+v(\{2,3\})>3$.

The theorem generalizes as follows:

THEOREM 2. The $n$-person game $v$ has an $(n-2)$-ply discriminatory solution $V$, discriminating against $\{3,4, \cdots, n\}$, with $\alpha_{3} \geqq \alpha_{4} \geqq \cdots$ $\geqq \alpha_{n}$, if and only if:

(a) $\alpha_{4}=\alpha_{5}=\cdots=\alpha_{n}=0$;

(b) $v(\{1,2\}) \geqq 1-\alpha_{3}$;

(c) If $\alpha_{3}>0$, then $v(\{1,3\})+v(\{2,3\})>1+2 \alpha_{3}$.

In this Case $\mathrm{V}$ consists of all imputations $\left(x_{1}, x_{2}, \alpha_{3}, 0, \cdots, 0\right)$ with $x_{1} \geqq 0, x_{2} \geqq 0, x_{1}+x_{2}=1-\alpha_{3}$.

Proof. The set of $\{1,2,3,4\}$-parts of $V$ must be a doubly discriminatory solution of the 4-person game $\bar{v}$, and hence Theorem 1 applies. As $\alpha_{4} \geqq \alpha_{5} \geqq \cdots \geqq \alpha_{n}$, it follows that $\alpha_{4}=\alpha_{5}=\cdots=\alpha_{n}=0$.

We must also show that $\{1,2,3,4\}$ is either effective or strong for the amount $q=1$. 
If $\alpha_{3}=0$, then $v(\{1,2\})=1$, so $v(\{1,2,3,4\})=1$ and $\{1,2,3,4\}$ is effective.

If $\alpha_{3}>0$, then $2 v(\{1,2\})+v(\{1,3\})+v(\{2,3\})>3$ but $v(\{1,2\})$ $\leqq 1$, so $v(\{1,2\})+v(\{1,3\})+v(\{2,3\})>2$ and this means the game $\bar{v}_{S, 1}$, where $S=\{1,2,3\}$ has no core. Hence $\{1,2,3\}$ and also $\{1,2,3,4\}$ is strong for $q \leqq 1$.

COROLlary. The game $v$ will have such a solution if and only if either $v(\{1,2\})=1$ or $2 v(\{1,2\})+v(\{1,3\})+v(\{2,3\})>3$.

A somewhat restricted form of this theorem was obtained, through substantially different methods, by Hebert in [2].

As a final corollary, we obtain a result which parallels that given by Gillies in [1]:

Corollary. A positive fraction of all n-person games has a common solution.

Proof. The common solution is simply the $(n-2)$-ply discriminatory solution given above (Theorem 2) with $\alpha_{3}$ small but positive. In fact, the only restrictions on $v$ are the inequalities given in the theorem, and these do not lower the dimension of the set.

\section{BIBLIOGRAPHY}

1. Donald B. Gillies, Solutions to general non-zero-sum games, Contributions to the Theory of Games, IV, Annals of Mathematics Studies No. 40, Princeton Univ. Press, Princeton, N. J., 1959, pp. 47-87.

2. Michael Hebert, The doubly discriminatory solutions of the four-person constantsum games, Advances in Game Theory, Annals of Mathematics Studies No. 50, Princeton Univ. Press, Princeton, N. J., 1964, pp. 345-377.

3. G. Owen, On a class of discriminatory solutions to simple games, Abstract 604-21, Notices Amer. Math. Soc. 11 (1964), 51.

4. Lloyd Shapley, Notes on $N$-person games. VI. On solutions that exclude one or more players, The Rand Corporation Research Memorandum RM-2533, 1960.

FORDHAM UNIVERSITY 